\title{
Correspondence: On the nature of strong piezoelectricity in graphene on $\mathrm{SiO}_{2}$
}

\author{
Christoph Stampfer ${ }^{1,2} \&$ Sven Reichardt ${ }^{1,3}$
}

\author{
Nature Communications 7:11570 doi: 10.1038/ncomms11570 (2016); Published 17 May 2016
}

Spatially resolved Raman spectroscopy and piezoresponse force microscopy are very interesting and useful tools for investigating properties of graphene and other two-dimensional materials. In a recent article published in Nature Communications, da Cunha Rodrigues et al. ${ }^{1}$ used both methods to investigate single-layer graphene deposited on $\mathrm{SiO}_{2}$ grating substrates. Interestingly, the authors report on strong piezoelectricity and on high in-plane strain values of $3-5 \%$ in the supported graphene regions. It is argued that the in-plane strain originates from the strong interaction of the carbon atoms with the oxygen atoms of the $\mathrm{SiO}_{2}$ substrate. Their finding of high in-plane strain is crucial, as it is considered to be of the same origin as the observed strong piezoelectricity in graphene on $\mathrm{SiO}_{2}$. Unfortunately however, a major correction is needed. The strain values reported by da Cunha Rodrigues et al. appear to be more than a factor 50 too large, that is, the actual strain in their investigated samples is only on the order of $0.06-0.10 \%$ or lower.

To extract the amount of strain in their samples, the authors employ confocal Raman spectroscopy, which has been shown to be a reliable tool for this purpose $\mathrm{s}^{2-5}$. The reported values of the Raman $\mathrm{G}$ band frequencies are within a reasonable and expected range for graphene supported by $\mathrm{SiO}_{2}$ (refs 6-8), with the average frequency on the supported region around $1,587 \mathrm{~cm}^{-1}$ and a frequency spread on the order of $1 \mathrm{~cm}^{-1}$. To convert these Raman shifts into strain values, the authors assume that the strain is of uniaxial nature and use a Grüneisen parameter of $\Delta \omega_{\mathrm{G}} / \Delta \varepsilon=-49.3 \mathrm{~cm}^{-1}=-0.493 \mathrm{~cm}^{-1} / \%$ (see page 6 in Supplementary Note 1 of da Cunha Rodrigues et al..$^{1}$ ). Correspondingly they extract strain values in the range of $3-5 \%$ in the supported region. However, the Grüneisen parameter taken from Bisset et al. ${ }^{9}$ (ref. 3 in Supplementary Note 1 of da Cunha Rodrigues et al. ${ }^{1}$ ) should read $\Delta \omega_{\mathrm{G}} / \Delta \varepsilon=-49.3 \mathrm{~cm}^{-1} / \%$ (see text in Bisset et $a .^{9}{ }^{9}$ ). Please note that in Table 1 of Bisset et al. ${ }^{9}$, there unfortunately is a mistake in the units: $\mathrm{cm}^{-1}$ should be replaced by $\mathrm{cm}^{-1} / \%$; the units are however fine in the text. By using the correct units, this leads to strain values which are exactly a factor 100 smaller than what has been claimed by the authors.

Moreover, using more established Grüneisen parameters for uniaxial strain, which range from -21.3 to $-23.5 \mathrm{~cm}^{-1} / \%$ (refs 2,10) and are in good agreement with the results of a first principles calculation ${ }^{2}$, results in strain values of $0.06-0.10 \%$, instead of the claimed $3-5 \%$. Furthermore, it is not clear whether the nature of the strain is uniaxial. Assuming biaxial strain instead, the extracted strain values are reduced by a further factor of 3 if one uses a Grüneisen parameter of $-69.1 \mathrm{~cm}^{-1} / \%$, as previously measured for biaxial strain ${ }^{5}$, which would result in an in-plane strain of $0.02-0.03 \%$ only.

In view of these corrected, smaller values of in-plane strain, we think the authors should reconsider their interpretation of the observed strong piezoelectricity. The authors themselves assume the origin of their reported high in-plane strain values to be the very same as the one of the strong piezoelectricity. The latter is attributed to non-zero net dipole moments in the system due to the chemical interaction between carbon and oxygen atoms, while at the same time the $\mathrm{C}-\mathrm{O}$ bonds are said to be the origin of the high in-plane strain values ${ }^{1}$.

However, except for the claimed very high in-plane strain, the paper offers no clear motivation of why carbon-oxygen bonds should form at the interface between graphene and the $\mathrm{SiO}_{2}$ substrate. While the authors do cite two theoretical studies ${ }^{11,12}$ to support their claim of strong covalent $\mathrm{C}-\mathrm{O}$ bonds, these studies investigate the highly idealized geometry of graphene on crystalline, $\alpha$-quartz $\mathrm{SiO}_{2}$. As also commented upon in these studies, the experimental situation significantly differs from this scenario, since the commonly used commercially available $\mathrm{Si} / \mathrm{SiO}_{2}$ wafers, as also used in the experiment by da Cunha Rodrigues et al. ${ }^{1}$, feature amorphous $\mathrm{SiO}_{2}$, on which graphene forms much weaker bonds. A more careful theoretical calculation $^{13}$, while still assuming crystalline $\mathrm{SiO}_{2}$, used non-cleaved, fully oxygen-terminated surfaces and found only weak bonds between carbon and oxygen atoms and only small dipole moments induced in the interfacial region. It should also be noted that the strength of the bonds is also influenced by the presence of $\mathrm{H}$-passivation of the oxygen dangling bonds, which further weakens the interaction between graphene and $\mathrm{SiO}_{2}$. The presence of strong $\mathrm{C}-\mathrm{O}$ bonds would also be at odds with the values of charge carrier mobility of graphene on $\mathrm{SiO}_{2}$ commonly found in the literature.

\footnotetext{
${ }^{1}$ JARA-FIT and 2nd Institute of Physics, RWTH Aachen University, 52074 Aachen, Germany. ${ }^{2}$ Peter Grünberg Institute (PGI-9), Forschungszentrum Jülich, 52425 Jülich, Germany. ${ }^{3}$ Physics and Material Science Research Unit, Université du Luxembourg, 1511 Luxembourg, Luxembourg. Correspondence and requests for materials should be addressed to C.S. (email: stampfer@physik.rwth-aachen.de).
} 
In conclusion, neither the reported Raman measurements nor the cited theoretical studies provide convincing proof for the authors' claim of strong covalent $\mathrm{C}-\mathrm{O}$ bonds being responsible for the observed behaviour in the piezoresponse force microscopy measurements, casting severe doubt on the schematic illustration in Fig. 6 of ref. 1, on which most of the discussion is based. As such, we encourage the authors to reconsider their argument of strong substrate-induced piezoelectricity in graphene caused by the formation of polar $\mathrm{C}-\mathrm{O}$ bonds, for which there appears to be no conclusive experimental evidence.

\section{References}

1. da Cunha Rodrigues, G. et al. Strong piezoelectricity in single-layer graphene deposited on $\mathrm{SiO}_{2}$ grating substrates. Nat. Commun. 6, 7572 (2015).

2. Mohiuddin, T. M. G. et al. Uniaxial strain in graphene by Raman spectroscopy: $G$ peak splitting, Grüneisen parameters, and sample orientation. Phys. Rev. B 79, 205433 (2009).

3. Ding, F. et al. Stretchable graphene: a close look at fundamental parameters through biaxial straining. Nano Lett. 10, 3453-3458 (2010).

4. Zabel, J. et al. Raman spectroscopy of graphene and bilayer under biaxial strain: bubbles and balloons. Nano Lett. 12, 617-621 (2012).

5. Lee, J. E., Ahn, G., Shim, J., Lee, Y. S. \& Ryu, S. Optical separation of mechanical strain from charge doping in graphene. Nat. Commun. 3, 1024 (2012).

6. Graf, D. et al. Spatially resolved Raman spectroscopy of single- and few-layer graphene. Nano Lett. 7, 238-242 (2007).

7. Pisana, S. et al. Breakdown of the adiabatic Born-Oppenheimer approximation in graphene. Nat. Mater. 6, 198-201 (2007).

8. Froehlicher, G. \& Berciaud, S. Raman spectroscopy of electrochemically gated graphene transistors: geometrical capacitance, electron-phonon, electron-electron, and electron-defect scattering. Phys. Rev. B 91, 205413 (2015).
9. Bisset, M. A., Izumida, W., Saito, R. \& Ago, H. Effect of domain boundaries on the Raman spectra of mechanically strained graphene. ACS Nano 6, 10229-10238 (2012).

10. Yoon, D., Son, Y.-W. \& Cheong, H. Strain-dependent splitting of the double-resonance Raman scattering band in graphene. Phys. Rev. Lett. 106, 155502 (2011).

11. Kang, Y.-J., Kang, J. \& Chang, K. J. Electronic structure of graphene and doping effect on $\mathrm{SiO}_{2}$. Phys. Rev. B 78, 115404 (2008).

12. Shemella, P. \& Nayak, S. K. Electronic structure and band-gap modulation of graphene via substrate surface chemistry. Appl. Phys. Lett. 94, 032101 (2009).

13. Nguyen, T. C., Otani, M. \& Okada, S. Semiconducting electronic property of graphene adsorbed on (0001) surfaces on $\mathrm{SiO}_{2}$. Phys. Rev. Lett. 106, 106801 (2011).

\section{Author contributions}

C.S. and S.R. discussed in detail ref. 1 and co-wrote the brief comment.

\section{Additional information}

Competing financial interests: The authors declare no competing financial interests.

Reprints and permission information is available online at http://npg.nature.com/ reprintsandpermissions/

How to cite this article: Stampfer, C. \& Reichardt, S. Correspondence: On the nature of strong piezoelectricity in graphene on $\mathrm{SiO}_{2}$. Nat. Commun. 7:11570 doi: 10.1038/ ncomms11570 (2016).

\section{(c) (1)}

This work is licensed under a Creative Commons Attribution 4.0 International License. The images or other third party material in this article are included in the article's Creative Commons license, unless indicated otherwise in the credit line; if the material is not included under the Creative Commons license, users will need to obtain permission from the license holder to reproduce the material. To view a copy of this license, visit http://creativecommons.org/licenses/by/4.0/ 